\title{
Soluble Interleukin 2 Receptor-Alpha (sIL-2R $\alpha)$ in the Peripheral Blood of Dogs-Comparison of Malignant Neoplasia with Other Diseases
}

\author{
Christian Prachar ${ }^{1}$, Franz-Josef Kaup ${ }^{2}$, Stephan Neumann ${ }^{{ }^{*}}$ \\ ${ }^{1}$ Small Animal Clinic, Institute of Veterinary Medicine, University of Goettingen, Goettingen, Germany \\ ${ }^{2}$ German Primate Centre, Goettingen, Germany

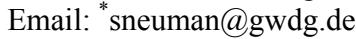

Received April 22, 2013; revised May 22, 2013; accepted June 9, 2013

Copyright (C) 2013 Christian Prachar et al. This is an open access article distributed under the Creative Commons Attribution License, which permits unrestricted use, distribution, and reproduction in any medium, provided the original work is properly cited.

\begin{abstract}
Background: Cytokines are mediators of diseases. Expression levels in the blood could be of clinical relevance. Objective: sIL-2R $\alpha$ is used as a marker for different malignancies in human medicine. The aim of this study was to show if sIL-2R $\alpha$ is detectable and if there is any correlation to different diseases in dogs. Methods: For this purposes sIL-2R $\alpha$ concentrations in the blood were measured in healthy dogs, in dogs with different non-neoplastic diseases and benign tumors and in dogs with malignant tumors. Serum levels of sIL-2R $\alpha$ were measured by using a human specific enzyme linked immunosorbent assay (ELISA). Results: Measurement of sIL-2R $\alpha$ was successful in most of the samples. Dogs with diseases have significantly increased serum levels of sIL-2R $\alpha$ compared to healthy controls. sIL-2R $\alpha$ serum levels are higher in patients with non-neoplastic diseases and benign tumors than in those with malignant neoplasia. There is a strong correlation between sIL-2R $\alpha$ and leukocyte count. Conclusion: Measurements of sIL-2R $\alpha$ in serum may be helpful in detecting stages and grades of inflammation in the progression of disease. sIL-2R $\alpha$ could actually not be used as an indicator for malignant diseases in dogs like in humans. The strong correlation between sIL-2R $\alpha$ and the leukocyte count indicates the inflammatory response to the disease. This could be helpful in giving a prognosis in some cases, because the inflammatory reaction is of prognostic relevance in different diseases including malignant and non-malignant neoplasia. Although the results of our research studies were very promising, further studies should be performed with a canine ELISA.
\end{abstract}

Keywords: sIL-2R $\alpha$; Soluble Interleukin-2 Receptor Alpha; ELISA; Dog

\section{Introduction}

Mature IL-2 molecule binds to the IL-2 receptor consisting of three subunits $(\alpha, \beta$ and $\gamma)$. Both $\beta$-(CD 122) and $\gamma$-subunit (CD 132) are already present on the cell surface. The $\alpha$-subunit is expressed when a specific antigen activates the target cell. Only when all three subunits are present on the cell binding affinity of IL-2 is high enough to bind to the receptor [1]. Expression of IL-2-receptor molecules is increased in activated T-cells. Furthermore, a soluble form of the receptor is released from the cell (sIL-2R $\alpha$ ). The soluble form of the IL-2 receptor consists of the $\alpha$-chain of the membrane-tied receptor.

T-lymphocytes play a central role in the immune system. They maintain cellular immunity, especially against intracellular pathogens (viruses, bacteria, parasites). The

"Corresponding author. binding of IL-2 on the cell surface triggers the main signal for T-lymphocyte activation. For the quantification of lymphocytic activation in humans the soluble IL-2 receptor alpha is used.

So sIL-2R $\alpha$ became an established marker for the monitoring of graft rejection after organ transplantation, especially regarding kidneys, hearts and livers: increased levels appear in case of graft rejection by the organ receiver [2-5], because those graft versus host reactions go along with T-lymphocyte activation.

In human medicine, sIL-2R $\alpha$ is also an already established marker for different diseases. In humans sIL-2R $\alpha$ is known to be expressed mainly by lymphoid cancer cells. A retrospective study from 2008 showed that expression of sIL-2R and its soluble form increased in most haematological malignancies, including different types of leukaemias and lymphomas [6]. Furthermore both lym- 
phoid and non-lymphoid tumor cells express more sIL$2 \mathrm{R} \alpha$. They include malignant melanoma, carcinomas of the kidney and different tumors of the head, neck an oesophagus as well as lung cancer [6,7]. There is a strong correlation between increased serum levels of SIL-2R $\alpha$ and the severity of disease in patients with a pulmonary sarcoidosis [8]. Since this correlation was found $\operatorname{sIL}-2 \mathrm{R} \alpha$ is used routinely as a marker for the diagnose and progression of sarcoidosis. In 2011 a study was made to find prognostic factors for B-cell lymphoma in humans. Increased serum levels of sIL-2R $\alpha$ were determined as very important prognostic factors for this disease [9].

Increased sIL-2R $\alpha$ serum levels were also found in patients with nasopharyngeal carcinomas [10], renal cell carcinomas [11] and autoimmune chronic active hepatitis [12]. One study demonstrated that dysregulated expression of IL-2 receptors in canine lymphoid and haematopoietic malignancies could be used as a model for human cancer [13]. Another study was made on human patients with systemic lupus erythematodes [14] which showed that in SLE patient sIL-2R $\alpha$ levels in serum and urine were increased.

So far no studies were made regarding stability and storage of sIL-2R $\alpha$ in canine blood samples. Besides there are neither studies to determine reference ranges for serum levels of sIL-2R $\alpha$ in healthy dogs.

To find out if the disease goes along with inflammation we measured the number of leukocytes in peripheral blood of the dogs tested on sIL-2R $\alpha$ serum levels. It was interesting to see if increased serum levels of sIL-2R $\alpha$ are correlated with leukocytosis in any way. Within the first 6 to 12 hours of inflammatory reaction leukocyte count increases and mature, so-called segmented neutrophil granulocytes are set free by the bone marrow. Bacterial infections, tumors and many other different diseases can lead to inflammation, too.

The aim of this study was to measure serum levels of sIL$2 \mathrm{R} \alpha$ in dogs and to find evidence if serum levels of sIL$2 \mathrm{R} \alpha$ in $\operatorname{dogs}$ are correlated with leukocytosis to find out if sIL-2R $\alpha$ was a possible marker for inflammatory diseases. Furthermore we analyzed if serum levels of sIL$2 \mathrm{R} \alpha$ differ in cases of different non-tumorous and tumorous diseases compared to dogs with malignant tumors. Based on the results of human studies we assumed that sIL-2R $\alpha$ may be increased in serum of dogs with tumors, especially in those with malignancies.

\section{Material and Methods}

\subsection{Animals}

Altogether serum levels of 36 dogs were measured (nonneoplastic diseases and benign neoplasia $\mathrm{n}=20$, malignnant neoplasia $n=16$ ). The control group consisted of 12 healthy individuals. 22 dogs were bitches (10 with ma- lignant neoplasia) and 14 male dogs (6 with malignant neoplasia). 16 individuals were crossbreed while 20 were breed dogs, most frequent breed were German shepherd (3) and boxer (3). The study was made according to the German animal welfare act.

Serum was isolated and aliquoted four times. The first time point was set to the time right after the isolation. The remaining three aliquots were frozen down immediately at $\leq-20^{\circ}$ Celsius. After 3, 6 and 9 weeks concentrations of sIL2-R $\alpha$ were measured.

\subsection{ELISA}

We used an ELISA developed for the quantitative determination of human sIL-2R $\alpha$ concentrations in serum (Quantikine ${ }^{\circledR}$ ELISA, catalog number DR2A00, R\&D Systems, Minnesota/USA). The genetic sequence homology of $77 \%$ regarding human and canine sIL-2R $\alpha$ made it possible to use a human ELISA to measure serum levels of sIL-2R $\alpha$ in canine blood samples.

ELISA technique is based on monoclonal antibody specific for sIL-2R $\alpha$ that has been pre-coated onto a microplate. The measurement of sIL-2R $\alpha$ was according to manufactures recommendation. To determine the optical density of each well a microplate reader set to $450 \mathrm{~nm}$ wavelength was used.

The dogs tested for serum levels of sIL-2R $\alpha$ were patients of the Small Animal Clinic of Georg-August-University in Goettingen/Germany. Depending on their diseases diagnosis was found by general examination, blood and urine tests, X-ray, ultrasound diagnostics, computed tomography, endoscopy, diagnostic laparoscopy and histopathology. In single patients who suffered from different diseases simultaneously the most severe diagnose was mentioned in this study.

\subsection{Statistics}

The statistical analysis was performed with the SPSSstatistics program (version 21, IBM, NY/USA). For purposes of analysis, dogs with sIL-2R $\alpha$ values $\leq 0 \mathrm{pg} / \mathrm{ml}$ were set at $0 \mathrm{pg} / \mathrm{ml}$. The dogs with diseases were divided into two groups: dogs with non-malignant diseases and dogs with malignancies. Furthermore it was tested if there was a correlation between sIL-2R $\alpha$ serum levels and the leukocyte count (reference range: $6-12 \mathrm{~K} / \mu \mathrm{l}$, all values $>12$ $\mathrm{K} / \mu \mathrm{l}$ are a so called leukocytosis).

Because both concentrations of sIL-2R $\alpha$ and the number of leukocytes were not normally distributed, nonparametric methods were used for statistical analysis.

To compare the sIL-2R $\alpha$ concentrations between patients with and without malignancies the Mann-Whitney $\mathrm{U}$ test was used. For the comparison of sIL-2R $\alpha$ in serum and leukocyte count Mann-Whitney U test was used, too. In all cases a p-value $<0.05$ was considered statistically 
significant.

\section{Results}

\subsection{Storage}

Our analyses showed that sIL2-R $\alpha$ in serum can be stored at $\leq-20^{\circ}$ Celsius for at least nine weeks. Even after that time there was no obvious decrease of $\operatorname{sIL} 2-\mathrm{R} \alpha$ in the samples.

\section{2. sIL-2R $\alpha$ in Healthy Dogs}

Significantly higher serum levels of sIL-2R $\alpha$ were found in patients suffering from disease compared to those of healthy controls (U-test $\mathrm{p}<0.001$ ).

The healthy control group consisted of 12 dogs. 7 dogs showed detectable amounts of sIL-2R $\alpha$ in serum, but only in low concentration ranges $(0.25-9 \mathrm{pg} / \mathrm{ml})$.

\section{3. sIL-2R $\alpha$ in Dogs with Different Diseases}

sIL-2R $\alpha$ serum concentrations were measured in $36 \mathrm{dogs}$ with different non-neoplastic diseases and benign neoplasia (20) and malignant neoplasia (16) (Table 1). Three out of the 14 male dogs showed no detectable sIL-2R in serum (21.4\%), and so did 4 of the 22 bitches (18.1\%).

Table 1. Serum levels of sIL-2R $\alpha$ in dogs with different malignant and non-malignant diseases (neg = concentration below detection limit). Numbers 1 - 20 different non-neoplastic diseases and benign neoplasia, 21 - 36 malignant neoplasia.

\begin{tabular}{|c|c|}
\hline Diagnosis & $\mathrm{sIL}-2 \mathrm{R} \alpha[\mathrm{pg} / \mathrm{ml}]$ \\
\hline 1) Acute renal failure & neg \\
\hline 2) Chronic renal failure & neg \\
\hline 3) Chronic renal failure, anaemia & 63.8 \\
\hline 4) Chronic renal failure & 58 \\
\hline 5) Liver cirrhosis & 104.4 \\
\hline 6) Haemorrhagic liver necrosis & 71.1 \\
\hline 7) Liver fibrosis, low-grade hepatitis & neg \\
\hline 8) Leishmaniasis & 856 \\
\hline 9) Leishmaniasis & 104.25 \\
\hline 10) Purulent rhinitis & 246 \\
\hline 11) Borreliosis, anaplasmosis, high anti-thrombocyte antibody titer & 54.2 \\
\hline 12) Diabetes mellitus & 45 \\
\hline 13) Diabetes mellitus & 29.8 \\
\hline 14) Diabetes mellitus, multiple organ failure & 22.2 \\
\hline 15) Pyometra, peritonitis & 46.2 \\
\hline 16) Pyometra & 5.4 \\
\hline 17) Pancreatitis with mesenterial steatonecrosis and fibrosing steatitis & 48.6 \\
\hline 18) Adenoma of hepatoid glands & 5.5 \\
\hline 19) Collagenous hamartoma, trichoepithelioma, pyogranulomatous dermatitis & 76.6 \\
\hline 20) Haemangioma of the spleen & 70.2 \\
\hline 21) Malignant lymphoma & 71.8 \\
\hline 22) Malignant lymphoma & neg \\
\hline 23) Malignant melanoma & 45.4 \\
\hline 24) Malignant melanoma & 36.4 \\
\hline 25) Haemangiosarcoma of the spleen & 43.6 \\
\hline 26) Haemangiosarcoma of the spleen & 27.6 \\
\hline 27) Intraductal papillary carcinoma of the mamma & 38.2 \\
\hline 28) Tubular carcinoma of the mamma & 35 \\
\hline 29) Complex carcinoma of the mamma with chondroid metaplasia & neg \\
\hline 30) Cystic granulosa-cell tumor, leiomyoma of the cervix & 67 \\
\hline 31) Adenocarcinoma, lymphangiosis carcionamtosa & neg \\
\hline 32) Testicular tumor (diffuse seminoma) & neg \\
\hline 33) Lymphoplasmacellular gastritis, neuroendocrine carcinoma & 57.2 \\
\hline 34) Prostate tumor with liver metastases & 30 \\
\hline 35) Renal adenocarcinoma & 10.2 \\
\hline 36) Haemangiopericytoma & 7 \\
\hline
\end{tabular}


The age of the dogs in the malignancy group lay a little higher than in the non-neoplastic/benign tumor group, in detail between 5 and 13 years in the malignant neoplasiagroup (median 10.5, mean 10.1) and between 3 and 11 years in the non-neoplastic/benign tumor group (median 9.5, mean 7.6).

The levels of 29 dogs with detectable sIL-2R $\alpha$ concentrations were between $5.4-856.0 \mathrm{pg} / \mathrm{ml}$. Median was $58.0 \mathrm{pg} / \mathrm{ml}$ in the group of non-neoplastic diseases/benign tumors (total range $5.4-865.0 \mathrm{pg} / \mathrm{ml}$ ) compared to a median of $37.3 \mathrm{pg} / \mathrm{ml}$ in the malignant neoplasia group (7.0 - $71.8 \mathrm{pg} / \mathrm{ml}$ ) (Figures 1-4). The overall mean in the non-malignant group $(112.2 \mathrm{pg} / \mathrm{ml})$ lay appreciably over the one in the malignant neoplasia group $(39.1 \mathrm{pg} / \mathrm{ml})$ (Figures 3 and 4).

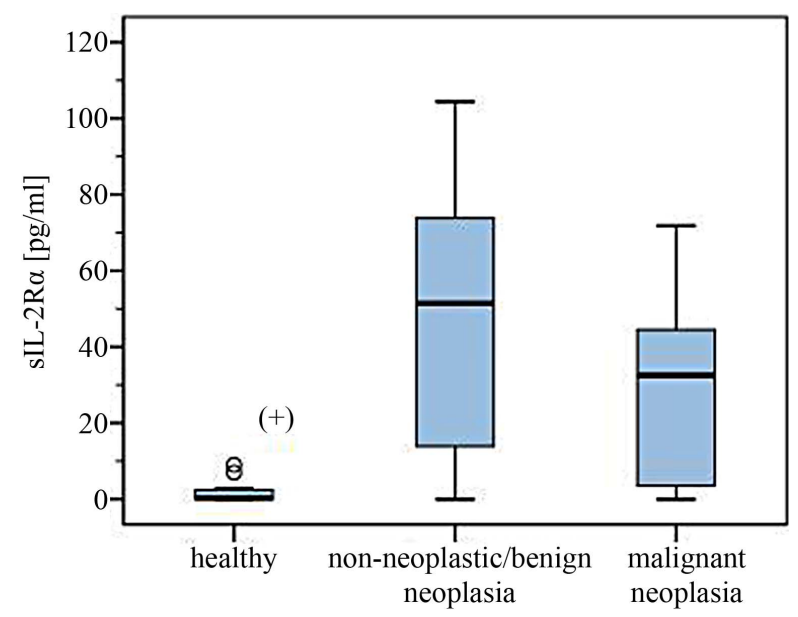

Figure 1. Serum levels of sIL-2R $\alpha$ in dogs with different non-neoplastic diseases/benign tumors and malignant neoplasia compared to healthy controls. $(+)$ Significantly lower serum levels in the healthy control group.

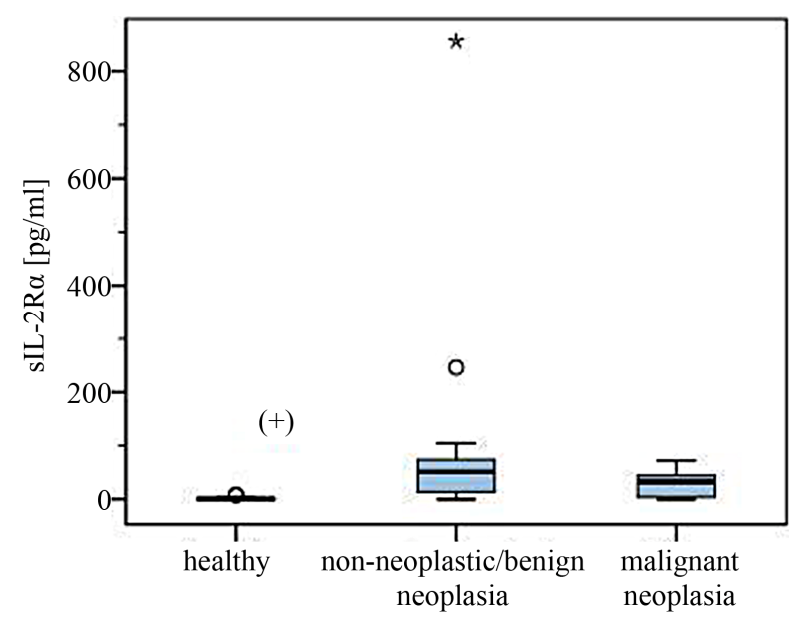

Figure 2. Serum levels of sIL-2R $\alpha$ in dogs with different non-neoplastic diseases or benign tumors and malignant neoplasia compared to healthy controls (with statistical outliers in the non-neoplastic/benign tumor group). (+) Significantly lower serum levels in the healthy control group.

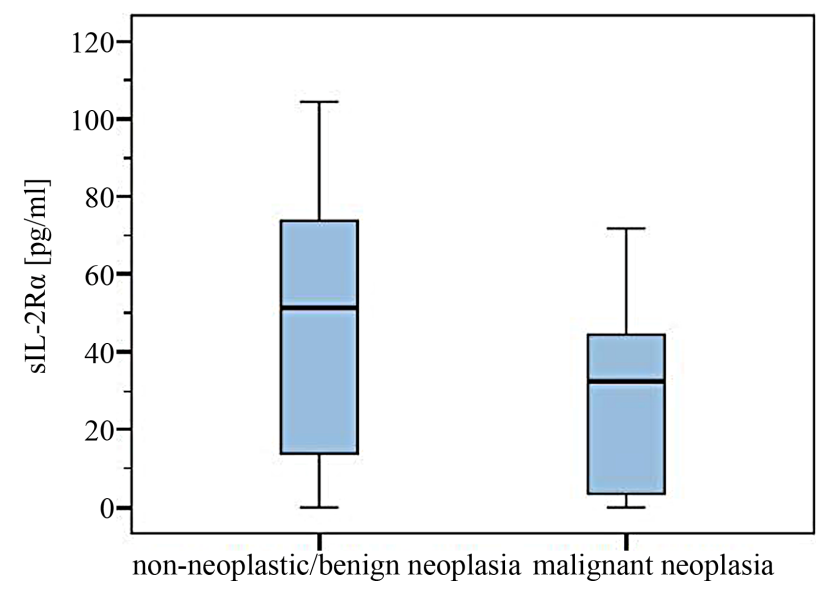

Figure 3. Comparison of sIL-2R $\alpha$ serum levels in dogs with and without malignant neoplasia.

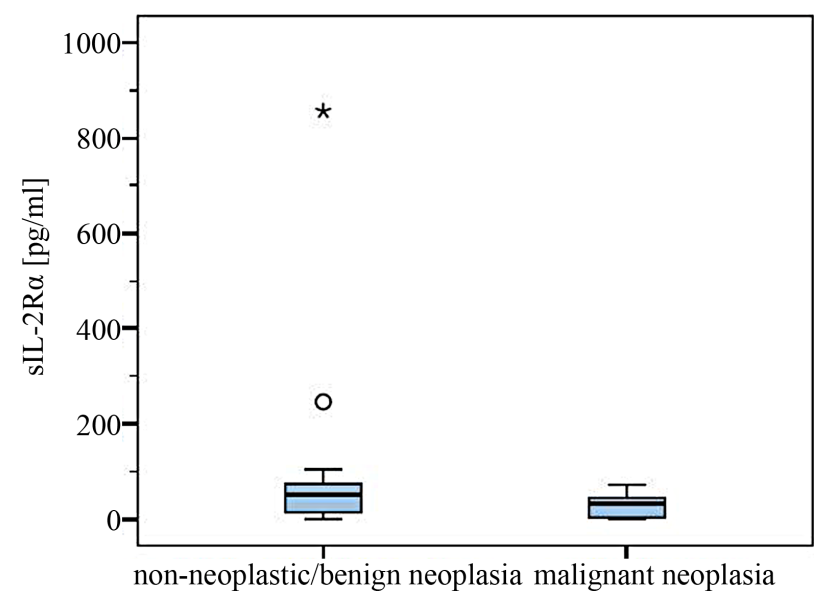

Figure 4. Comparison of sIL-2R $\alpha$ serum levels in dogs with and without malignant neoplasia (with statistical outliers in the non-neoplastic/benign tumor group).

In the malignant neoplasia group 3 dogs were tested negatively on sIL-2R $\alpha$, while in the other group $4 \operatorname{dogs}$ had no detectable amount of sIL-2R $\alpha$ in serum.

Highest serum levels of sIL-2R $\alpha$ were all found in the malignant neoplasia group, in detail in a dog with leishmaniasis $(856 \mathrm{pg} / \mathrm{ml})$, one with a purulent rhinitis (246 $\mathrm{pg} / \mathrm{ml})$ and one with liver cirrhosis $(104.4 \mathrm{pg} / \mathrm{ml})$ (Figures 2 and 4). The highest serum level in the malignnant neoplasia group had one dog with a malignant lymphoma. Its serum level of $71.8 \mathrm{pg} / \mathrm{ml}$ lay clearly below the highest serum levels measured in the non-neoplastic/benign tumor group.

Serum levels of sIL-2R $\alpha$ compared to the number of leukocytes in peripheral blood 15 out of the group of 29 dogs tested positively on sIL-2R $\alpha$ showed a leukocytosis (leukocyte count $>12 \mathrm{~K} / \mu \mathrm{l}$ ) in peripheral blood. Together with the 7 dogs that had no detectable amount of sIL-2R $\alpha$ in serum (3 out of 7 with a leukocytosis) a correlation was found between sIL-2R $\alpha$ serum levels and the number 
of leukocytes (Mann-Whitney-U-test $\mathrm{p}<0.001$ ).

When we look at the two different disease groups it is noticeable that in always both groups a correlation was found between serum levels of sIL-2R $\alpha$ and the leukocyte count (Mann-Whitney-U-test $\mathrm{p}<0.05$ ).

Furthermore we tested two different groups of the patients suffering from disease regarding the amount of sIL-2R $\alpha$ and the number of leukocytes. In the non-malignancy group 17 out of the 20 dogs were tested positively on sIL-2R $\alpha$ in serum. 9 of them had a leukocytosis in peripheral blood.

In the malignant neoplasia group 12 out of 16 dogs had detectable amounts of sIL-2R $\alpha$ in blood while 6 of them showed a leukocytosis.

\section{Discussion}

Many diseases lead to immune activation with increased serum levels of sIL2-R $\alpha$. Both non-tumorous and tumorous diseases may go along with immune activation and inflammation. Data of many human studies suggest that depending on the character of the disease and the stage of inflammation sIL2-R $\alpha$ levels in peripheral blood may increase stronger following tumor growth as they do in any other disease, especially in malignant neoplasia [6, 9,15-17].

As mentioned above so far no studies were made to detect stability of sIL-2R $\alpha$ in stored canine blood samples, also no studies were made to determine reference ranges of sIL-2R $\alpha$ in healthy dogs. Results of our study assume that healthy dogs show very low serum levels but in case of inflammatory diseases the amount of measureable sIL-2R $\alpha$ increases. The absolute amount of detectable sIL-2R $\alpha$ in canine serum samples is appreciably lower than in most of the human studies. In most of the studies ranges of sIL-2R $\alpha$ levels in serum in healthy controls were $<2000 \mathrm{pg} / \mathrm{ml}$, based on the techniques and reference ranges of the different laboratories.

Whenever patients suffering from disease showed increased amounts of sIL-2R $\alpha$, levels were clearly higher than those of the healthy controls, for example in studies on patients with different tumors, autoimmune diseases and visceral leishmaniosis $[6,9,18,19]$.

Looking at our results there is a remarkable difference between serum levels in dogs with non-neoplastic disease and benign tumors compared to malignant neoplasia. The separation of the dogs into two groups was made according to the disease's character. In malignant diseases an early diagnosis enhances the chances for a successful therapy and may so lead to a better prognosis. Some retrospective studies showed that cancer is one of the most important natural death causes of dogs. Studies of lifetime risk have suggested a wide range in estimates of deaths due to cancer. Death rates went from $3 \%$ of deaths in military working German shepherds [20] to $27 \%$ of dogs from a German study [21]. In one study from the UK $16 \%$ of deaths were attributed to cancer, twice as many as to heart disease [22].

In our study highest serum levels were found in the group of non-neoplastic diseases and benign tumors that went along with inflammation. The absolute amounts of the patients with higher serum levels lay appreciably over those measured in the dogs with malignant neoplasia.

It is an interesting fact that sIL2-R $\alpha$ is an established marker in human medicine for diagnosis and progression of many tumorous diseases $[6,8,9,23]$, but does not have huge relevance as a marker for inflammation in non-tumorous diseases. There are two exceptions: rheumatoid arthritis [24] and human visceral leishmaniasis [18]. In human leishmaniasis increased serum levels of sIL-2R $\alpha$ were correlated with severity of disease and returned to the normal range during successful chemotherapy. In our study absolute highest amount of detectable sIL-2R $\alpha$ $(856.0 \mathrm{pg} / \mathrm{ml})$ and also the fourth-highest amount $(104.25$ $\mathrm{pg} / \mathrm{ml}$ ) were also found in dogs with leishmaniasis. Both serum levels were higher than those in the malignancy group. Leishmaniasis is rare in dogs in Germany, so it could be interesting to make further studies on dogs in countries where leishmaniasis appears to be a bigger problem.

Three dogs with diabetes mellitus were tested positively on sIL-2R $\alpha$ with similar serum levels between 22.2 and $45.0 \mathrm{pg} / \mathrm{ml}$. Actually the character of type 1 and type 2 diabetes mellitus as chronic inflammatory diseases with T-cell-mediated immune activation is discussed [25]. Type 1 diabetes mellitus is the main type of diabetes in dogs. It goes along with a loss of insulin-producing $\beta$-cells of the pancreas. Early stages of the disease are based on the secretion of high levels of pro-inflammatory cytokines. Those effects trigger the fatal destruction process of $\beta$ cells. There are approaches of anti-inflammatory interventions in treating type 1 diabetes mellitus in humans [26]. The three dogs with diabetes mellitus in our study were tested positively on sIL-2R $\alpha$ with similar serum levels $(22.2-45.0 \mathrm{pg} / \mathrm{ml})$. One of the dogs $(45.0 \mathrm{pg} / \mathrm{ml})$ showed clinical signs of acute renal failure and another one was suspected to have a pancreatitis $(29.8 \mathrm{pg} / \mathrm{ml})$. These data suggest that in dogs before receiving therapy to acute exacerbation of diabetes reactions of the immune system lead to increased sIL-2R $\alpha$. We can't be certain that only diabetes leads to increased sIL-2R $\alpha$ because two dogs also had other clinical signs following final stages of diabetes that could have the same effect, too.

Two of the dogs diagnosed with chronic renal failure showed similar serum levels of 58.0 and $63.8 \mathrm{pg} / \mathrm{ml}$, while one of them had no detectable sIL-2R $\alpha$ in serum. All three dogs had severe clinical symptoms and were 
euthanized within a couple of days after beginning of their treatment. In human studies it was shown that chronic renal failure is a major cause for T-lymphocyte activation, which leads to increased expression of sIL-2R $\alpha[27,28]$. Serum levels of sIL-2R $\alpha$ were significantly higher in patients with chronic renal failure compared to healthy controls [29]. Because early diagnosis and proper treatment of chronic renal failure can lead to a longer survival time maybe sIL-2R $\alpha$ could become a prognostic marker together with the already established diagnostic techniques.

Two of the dogs in the malignant neoplasia group were diagnosed with malignant lymphoma. In contrast to human medicine, where sIL-2R $\alpha$ is an already established marker for malignant lymphoma $[9,16,23]$, we could not find evidence that sIL-2R $\alpha$ is a useful marker for detecting malignant lymphoma in dogs. Although there were only two dogs with malignant lymphoma it is noticeable that only one of the dogs showed detectable sIL-2R $\alpha$ in serum: it was the highest serum level of the dogs in the malignant neoplasia group $(71.8 \mathrm{pg} / \mathrm{ml})$ which lay remarkably lower than the highest serum levels in the nonneoplastic/benign tumor group. But in addition it is an interesting fact that the malignant lymphoma patient with the reduced general condition and severe clinical symptoms was the one that had no detectable sIL-2R $\alpha$ in serum.

It is interesting that all three German shepherds belonged to the malignant neoplasia group and showed highly malignant tumors with poor prognosis (renal adenocarcinoma, haemangiosarcoma of the spleen, malignnant prostate tumor with liver metastases). Serum levels of sIL-2R $\alpha$ were ranged in relatively low amounts (10.2 $30.0 \mathrm{pg} / \mathrm{ml}$ ) compared to both the rest of the same group and the non-neoplastic/benign tumor group.

To find out if the disease goes along with inflammation we measured the number of leukocytes in peripheral blood of the dogs tested on sIL-2R $\alpha$ serum levels. It was interesting to see if increased serum levels of sIL-2R $\alpha$ are correlated with leukocytosis in any way. Within the first 6 to 12 hours of inflammatory reaction leukocyte count increases and mature, so-called segmented neutrophil granulocytes are set free by the bone marrow. Bacterial infections, tumors and many other different diseases can lead to inflammation, too. The number of leukocytes in peripheral blood is an already established marker for inflammation both in human and veterinary medicine since decades. Beside new markers for the grade of inflammation like C-reactive protein it was interesting to measure serum levels of sIL-2R $\alpha$ to see if there was a correlation between those levels and the number of leukocytes.

Our data suggest that in dogs sIL2-R $\alpha$ is increased in acute inflammation whether in non-neoplastic and benign disease or in malignant neoplasia. Results show a ten- dency that sIL-2R $\alpha$ in dogs seems to be increased rather in non-neoplastic and benign diseases going along with inflammation.

In our study no evidence was found that malignant tumors automatically lead to significantly increased sIL$2 \mathrm{R} \alpha$ serum levels. This result noticeably differs from many of the human studies. Furthermore the results of our study show that there is a correlation between increased levels of sIL-2R $\alpha$ and the number of leukocytes in peripheral blood, and this correlation obviously does not depend on the character of the disease: correlation was found in both groups, whether non-neoplastic/benign tumor or malignant neoplasia.

The results of this study assume that sIL-2R $\alpha$ serum levels could be used as a pro-inflammatory diagnostic marker associated with different diseases. High serum levels of sIL-2R $\alpha$ in dogs seem to be related rather to non-neoplastic disease or benign tumors than to malignant neoplasia, though immune response in many malignant diseases also leads to increased sIL-2R $\alpha$ serum levels. Although there was no statistical significance found, there is an obvious tendency that sIL-2R $\alpha$ could be a helpful marker for the differentiation between non-neoplastic disease/benign tumor and malignant neoplasia. Further studies on a larger group of patients should follow to approve these results. Maybe another correlation can be found, for example between high sIL-2R $\alpha$ serum levels and an increased concentration of pro-inflammatory markers like C-reactive protein (CRP) in blood, which was not part of this study.

As a result sIL-2R $\alpha$ could become a useful diagnostic parameter for the detection of the moment and the degree of inflammatory response in the body.

Altogether measured amounts of sIL-2R $\alpha$ even in dogs suffering from the disease were noticeably lower than in human patients. Possibly a specific ELISA for the detection of canine sIL-2R $\alpha$ could lead to better results regarding the quantity of measurable serum levels. Though the sequence homology of $77 \%$ between human and canine sIL-2R $\alpha$ made it possible to use a human ELISA for detecting canine sIL-R $\alpha$ in serum samples, the absolute amounts of sIL-2R $\alpha$ could have been even higher than our results suggest.

Further studies should approve that sIL-2R $\alpha$ serum levels in dogs are significantly higher in diseases not caused by malignant neoplasia. sIL-2R $\alpha$ measurements in serum could become a marker for the differentiation if inflammation is caused by a malignant tumor or not. Interestingly, this would stay in contrast to many studies implemented in human medicine. In addition to that positive results of studies on a larger group of patients may give a useful hint if sIL-2R $\alpha$ can be used for the differentiation if inflammation is caused by a tumor or not, especially because both diagnosing and treating tumorous 
diseases becomes more and more important in small animal medicine.

\section{REFERENCES}

[1] I. R. Tizard, "Veterinary Immunology," Saunders, St. Louis/Missouri, 2008.

[2] R. K. Gupta, M. Jain and R. K. Sharma, "Serum \& Urinary Interleukin-2 Levels as Predictors in Acute Renal Allograft Rejection," Indian Journal of Medical Research, Vol. 119, No. 5, 2004, p. 24-27.

[3] P. H. Lee, et al., "Serum Interleukin-2 and Soluble Interleukin-2 Receptor in Renal Transplant Recipients," Journal of the Formosan Medical Association, Vol. 91, No. 9, 1992, pp. 844-848.

[4] R. Di Stefano, et al., "Interleukin-2 Receptor Levels in End-Stage Renal Disease and Transplant Patients," Transplant Proceedings, Vol. 22, No. 4, 1990, pp. 1863-1864.

[5] G. C. Zucchelli, et al., "Increased Circulating Concentrations of Interleukin 2 Receptor during Rejection Episodes in Heart- or Kidney-Transplant Recipients," Clinical Chemistry, Vol. 36, No. 12, 1990, pp. 2106-2109.

[6] E. Bien and A. Balcerska, "Serum Soluble Interleukin 2 Receptor Alpha in Human Cancer of Adults and Children: A Review," Biomarkers, Vol. 13, No. 1, 2008, pp. 1-26. doi:10.1080/13547500701674063

[7] N. El Houda Agueznay, et al., "Soluble Interleukin-2 Receptor and Metalloproteinase-9 Expression in Head and Neck Cancer: Prognostic Value and Analysis of Their Relationships," Clinical and Experimental Immunology, Vol. 150, No. 1, 2007, pp. 114-123.

doi:10.1111/j.1365-2249.2007.03464.x

[8] E. Bargagli, et al., "Chitotriosidase and Soluble IL-2 Receptor: Comparison of Two Markers of Sarcoidosis Severity," Scandinavian Journal of Clinical \& Laboratory Investigation, Vol. 68, No. 6, 2008, pp. 479-483. doi:10.1080/00365510701854975

[9] Z. Z. Yang, et al., "Soluble IL-2Ralpha Facilitates IL-2Mediated Immune Responses and Predicts Reduced Survival in Follicular B-Cell Non-Hodgkin Lymphoma," Blood, Vol. 118, No. 10, 2011, pp. 2809-2820. doi:10.1182/blood-2011-03-340885

[10] S. H. Hsiao, et al., "Clinical Significance of Measuring Levels of Tumor Necrosis Factor-Alpha and Soluble Interleukin-2 Receptor in Nasopharyngeal Carcinoma," Acta Otolaryngology, Vol. 129, No. 12, 2009, pp. 1519-1523. doi:10.3109/00016480902849427

[11] T. Matsumoto, et al., "Serum Levels of Soluble Interleukin-2 Receptor in Renal Cell Carcinoma," Urology, Vol. 51, No. 1, 1998, pp. 145-149. doi:10.1016/S0090-4295(97)00476-7

[12] A. Lobo-Yeo, et al., "Soluble Interleukin 2 Receptors in Autoimmune Chronic Active Hepatitis," Gut, Vol. 31, No. 6, 1990, pp. 690-693. doi:10.1136/gut.31.6.690

[13] E. B. Dickerson, et al., "Potential to Target Dysregulated Interleukin-2 Receptor Expression in Canine Lymphoid and Hematopoietic Malignancies as a Model for Human Cancer," Journal of Immunotherapy, Vol. 25, No. 1, 2002, pp. 36-45. doi:10.1097/00002371-200201000-00004

[14] B. Brugos, et al., "Serum and Urinary Cytokine Levels of SLE Patients," Pharmazie, Vol. 67, No. 5, 2012, pp. 411413.

[15] A. M. Berghella, et al., "The Significance of an Increase in Soluble Interleukin-2 Receptor Level in Colorectal Cancer and Its Biological Regulating Role in the Physiological Switching of the Immune Response Cytokine Network from TH1 to TH2 and Back," Cancer Immunology, Immunotherapy, Vol. 45, No. 5, 1998, pp. 241-249. doi: $10.1007 / \mathrm{s} 002620050439$

[16] T. Akahane, et al., "Serum Soluble Interleukin-2 Receptor Levels in Patients with Malignant Lymphoma of Bone," Journal of Orthopaedic Science, Vol. 14, No. 3, 2009, pp. 248-252. doi:10.1007/s00776-009-1335-x

[17] A. Ottaiano, et al., "Soluble Interleukin-2 Receptor in Stage I-III Melanoma," Cytokine, Vol. 33, No. 3, 2006, pp. 150155. doi:10.1016/j.cyto.2006.01.002

[18] G. Vitale, et al., "The Significance of Serum Soluble IL-2 Receptor as a Marker for Active Visceral Leishmaniasis in Sicilian Patients," Clinical and Experimental Immunology, Vol. 90, No. 2, 1992, pp. 219-222. doi:10.1111/j.1365-2249.1992.tb07932.x

[19] D. Dejica, "Serum Soluble IL-2 Receptor as a Marker of Lymphocyte Activation in Some Autoimmune Diseases. Effect of Immunosuppressive Therapy," Roumanian Archives of Microbiology and Immunology, Vol. 60, No. 3, 2001, pp. 183-201.

[20] M. R. Peterson, R. A. Frommelt and D. G. Dunn, “A Study of the Lifetime Occurrence of Neoplasia and Breed Differences in a Cohort of German Shepherd Dogs and Belgian Malinois Military Working Dogs That Died in 1992," Journal of Veterinary Internal Medicine, Vol. 14, No. 2, 2000, pp. 140-145. doi:10.1111/j.1939-1676.2000.tb02227.x

[21] H. Eichelberg and R. Seine, "Lebenserwartung und Todesursachen bei Hunden-I. Zur Situation bei Mischlingen und Verschiedenen Rassen," Berliner und Munchener Tierarztliche Wochenschrift, Vol. 109, No. 8, 1996, pp. 292303.

[22] A. R. Michell, "Longevity of British Breeds of Dog and Its Relationships with Sex, Size, Cardiovascular Variables and Disease," Veterinary Record, Vol. 145, No. 22, 1999, pp. 625-629. doi:10.1136/vr.145.22.625

[23] T. Morito, et al., "Serum Soluble Interleukin-2 Receptor Level and Immunophenotype Are Prognostic Factors for Patients with Diffuse Large B-Cell Lymphoma," Cancer Science, Vol. 100, No. 7, 2009, pp. 1255-1260. doi:10.1111/j.1349-7006.2009.01167.x

[24] A. M. Witkowska, "On the Role of sIL-2R Measurements in Rheumatoid Arthritis and Cancers," Mediators of Inflammation, Vol. 2005, No. 3, 2005, pp. 121-130. doi:10.1155/MI.2005.121

[25] M. S. Akash, K. Rehman and S. Chen, "Role of Inflammatory Mechanisms in Pathogenesis of Type 2 Diabetes Mellitus," Journal of Cellular Biochemistry, Vol. 114, No. 3, 2012, pp. 525-531.

[26] B. Baumann, H. H. Salem and B. O. Boehm, "Anti-In- 
flammatory Therapy in Type 1 Diabetes," Current Diabetes Reports, Vol. 12, No. 5, 2012, pp. 499-509.

doi:10.1007/s11892-012-0299-y

[27] C. J. Kelly, "T Cell Function in Chronic Renal Failure and Dialysis," Blood Purif, Vol. 12, No. 1, 1994, pp. 3641. doi:10.1159/000170143

[28] K. H. Shu, et al., "Soluble Interleukin 2 Receptor in Dia- lyzed Patients," Artif Organs, Vol. 22, No. 2, 1998, pp. 142-144. doi:10.1046/j.1525-1594.1998.05062.x

[29] H. Sugimoto, et al., "The Clinical Significance of the Measurement of Serum Soluble Interleukin-2 Receptors in Various Diseases," Rinsho Byori, Vol. 44, No. 2, 1996, pp. 176-182. 